\title{
Abstract from Current Literature
}

Circulating cardiac troponins levels and cardiac dysfunction in children with acute and fulminant viral myocarditis

Mohammed Al-Biltagi ${ }^{1}$, Mona Issa ${ }^{2}$, Hamdi Abo Hagar $^{1}$, Maher Abdel-Hafez ${ }^{1}$, Nahla Abdul Aziz ${ }^{3}$

Acta Paediatrica Volume 99, Issue 10, pages 15101516, October 2010

Objectives: To study the correlation between cardiac Troponins blood levels and degrees of cardiac dysfunction in children with acute and fulminant viral myocarditis and to study their prognostic role in predicting the outcomes and risk of having dilated cardiomyopathy.

Methodology: Troponin I \& T blood levels were measured in 65 children with acute or fulminant viral myocarditis. The cardiac functions of RV \& LV were assessed by Doppler echocardiography.

Results: The levels of cTnl \& CTnT were significantly higher in patients with fulminant myocarditis than in controls and children with acute myocarditis $(p<0.05$ $\&<0.001 *$ respectively). The cardiac functions were significantly impaired in fulminant myocarditis than in acute myocarditis $\left(p<0.001^{*}\right)$. There were negative correlations between the cardiac troponins levels and the cardiac functions measured by echocardiography in children with acute and fulminant myocarditis. There were 3 deaths (7.5\%), and 10 (25\%) children developed dilated cardiomyopathy in acute myocarditis while there were eight deaths (32\%) and one patient (4\%) who developed dilated cardiomyopathy in fulminant myocarditis group.

Conclusion: Cardiac troponins levels can predict the severity of myocarditis and the prognosis on the shortterm level. Fulminant myocarditis was associated with higher levels of both cTn I \& cTn T than acute myocarditis. Despite that fulminant myocarditis has a more aggressive course, the risk of developing cardiomyopathy was less than in acute myocarditis.

Keywords: Acute myocarditis; Cardiac troponin I; Cardiac troponin T; Children; Fulminant myocarditis

\section{Adrenal insufficiency after glucocorticoid withdrawal in children with rheumatic diseases}

Benedikt $\mathrm{M}$ Huber $^{1}$, Isabel $\mathrm{B}$ Bolt ${ }^{2}$, Marie-Josèphe Sauvain ${ }^{2}$, Christa E Flück ${ }^{1}$

Acta PaediatricaVolume 99, Issue 12, pages 18891893, December 2010

Aim: Glucocorticoids (GCs) are often used for the treatment of rheumatic disorders. However, doses are prescribed, which may suppress the hypothalamicpituitary-adrenal (HPA) axis. After GC withdrawal, recovery of the HPA axis may be delayed putting the patient at risk for adrenal insufficiency. We assessed adrenal function and factors influencing adrenal responsiveness after termination of GC therapy in paediatric patients with rheumatic diseases.

Methods: Nineteen patients aged 2-15 years were followed clinically, and adrenal function was tested by low-dose adrenocorticotropic hormone test 1 month after GC withdrawal. In case of adrenal insufficiency by test, re-assessment was performed after 6 and 18 months.

Results: No signs or symptoms of adrenal insufficiency occurred in any of the patients during and after GC withdrawal. Biochemical examination revealed adrenal insufficiency in 32\% (6/19) at 4 weeks and in $11 \%(2 / 19)$ at 20 months after GC withdrawal.

Conclusions: In conclusion, current strategies to withdraw GC from paediatric patients with rheumatic diseases are safe. Routine adrenal function testing after GC therapy and withdrawal may not be needed considering the low risk but high number of patients treated with GCs. Nevertheless, awareness of the potential risk and information of patients and their caregivers are crucial to avoid adrenal crisis.

Keywords: Adrenal insufficiency; Glucocorticoid withdrawal; Low-dose ACTH test; Paediatric rheumatology 
High Mortality Rates for Very Low Birth Weight Infants in Developing Countries Despite Training

Waldemar A. Carlo, MD ${ }^{a, b}$, Shivaprasad S. Goudar, MD, MHPE ${ }^{c}$, Imtiaz Jehan, FCPS, MSc ${ }^{d}$, Elwyn Chomba, MD ${ }^{\mathrm{b}, \mathrm{e}}$, Antoinette Tshefu, MD, $\mathrm{PhD}^{\mathrm{f}}$, Ana Garces, MDg et al.

PEDIATRICS Vol. 126 No. 5 November 2010, pp. e1072-e1080

Objective: The goal was to determine the effect of training in newborn care and resuscitation on 7-day (early) neonatal mortality rates for very low birth weight (VLBW) infants. The study was designed to test the hypothesis that these training programs would reduce neonatal mortality rates for VLBW infants.

Methods: Local instructors trained birth attendants from 96 rural communities in 6 developing countries in protocol and data collection, the World Health Organization Essential Newborn Care (ENC) course, and a modified version of the American Academy of Pediatrics Neonatal Resuscitation Program (NRP), by using a train-the-trainer model. To test the impact of ENC training, data on infants of 500 to $1499 \mathrm{~g}$ were collected by using a before/after, active baseline, controlled study design. A clusterrandomized, controlled trial design was used to test the impact of the NRP.

Results: A total of 1096 VLBW (500-1499 g) infants were enrolled, and $98.5 \%$ of live-born infants were monitored to 7 days. All-cause, 7-day neonatal mortality, stillbirth, and perinatal mortality rates were not affected by ENC or NRP training.

Conclusions: Neither ENC nor NRP training of birth attendants decreased 7-day neonatal, stillbirth, or perinatal mortality rates for VLBW infants born at home or at first-level facilities. Encouragement of delivery in a facility where a higher level of care is available may be preferable when delivery of a VLBW infant is expected.
Paracetamol in early infancy: the risk of childhood allergy and asthma

Egil Bakkeheim ${ }^{1}$, Peter Mowinckel ${ }^{1}$, Kai Håkon Carlsen $^{2,3}$, Geir Håland ${ }^{1}$, Karin C Lødrup Carlsen ${ }^{1,2}$

Acta Paediatrica Volume 100, Issue 1, pages 90-96, January 2011

Aim: We investigated whether paracetamol exposure in pregnancy and until 6 months of age was associated with allergic disease in school children.

Methods: In a prospective birth cohort study in Oslo, 1016 children included at birth were re-investigated at 10 years. Paracetamol exposure in pregnancy and until 6 months of age was registered. Outcomes at 10 years included current asthma, a history of asthma, allergic sensitization and allergic rhinitis.

Results: Maternal paracetamol use in the first trimester increased the risk for allergic rhinitis at 10 years OR (odds ratio) $(95 \% \mathrm{Cl}) 2.30(1.06,4.97)$ in boys and girls. Paracetamol use until 6 months in girls increased the risk for allergic sensitization OR $2.20(1.15,4.22)$ and a history of asthma OR $2.20(1.13,4.30)$. The ORs for allergic sensitization and history of asthma in girls remained unchanged adjusting for upper or lower airway infections during the first 6 months of life.

Conclusion: Paracetamol exposure in pregnancy was associated with allergic rhinitis, but not with asthma or allergic sensitization at 10 years of age. Paracetamol used until 6 months of age was associated with allergic sensitization and having a history of asthma in girls at 10 years of age, even considering concomitant airway infections. 